\title{
Risk of osteoporosis and fracture in long-term breast cancer survivors
}

Jieon Go 1 , Suyeon Park', Kyeong Sik Kim ${ }^{1}$, Min Chang Kang', Myong Hoon Ihn', Sangchul Yun', Sang Hyun Kim', Sung Hoon Hong ${ }^{3}$, Jong Eun Lee ${ }^{3}$, Sun Wook Han ${ }^{3}$, Sung Yong Kim³ ${ }^{3}$ Zisun Kim ${ }^{4}$, Sung Mo Hur ${ }^{4}$, Jihyoun Lee ${ }^{1}$

'Department of Surgery, Soonchunhyang University Seoul Hospital, Seoul;

${ }^{2}$ Department of Biostatistics, Soonchunhyang University College of Medical Sciences, Seoul;

${ }^{3}$ Department of Surgery, Soonchunhyang University Cheonan Hospital, Cheonan;

${ }^{4}$ Department of Surgery, Soonchunhyang University Bucheon Hospital, Bucheon, Korea

Purpose: High incidence of osteoporosis has been reported in breast cancer patients due to early menopause triggered by adjuvant treatment and temporary ovarian function suppression. In this study, we sought to determine whether long-term breast cancer survivors had an elevated risk of low bone density compared to the general population.

Methods: Long-term breast cancer survivors who had been treated for more than 5 years were selected for this study. Data were obtained from medical records and using a questionnaire from the Korea National Health and Nutrition Examination Survey (KNHANES). An agematched non-cancer control group was selected from the KNHANES records. Incidence of fracture and bone mineral density (BMD) were compared between the two groups.

Results: In total, 74 long-term breast cancer survivors and 296 non-cancer controls were evaluated. The incidence of fracture did not differ between the two groups $(P=0.130)$. No differences were detected in lumbar BMD $(P=0.051)$ following adjustment for body mass index, while hip BMD was significantly lower in breast cancer survivors $(P=0.028)$. Chemotherapy and endocrine treatment were not related to low BMD in breast cancer survivors. In more than half of the survivors, the 10-year risk of osteoporotic fracture was less than 1\%.

Conclusion: Long-term breast cancer survivors had low bone density but a comparable risk of fracture compared to non-cancer agematched controls. Further studies on the factors related to low bone density in long-term breast cancer survivors are required.

Keywords: Breast neoplasms, Survivorship, Osteoporosis, Osteoporotic fracture

Received: May 6, 2020 Revised: Jun 17, 2020 Accepted: Jun 22, 2020

Correspondence to: Jihyoun Lee

Department of Surgery, Soonchunhyang University Seoul Hospital,

59 Daesagwan-ro, Yongsan-gu, Seoul 04401, Korea

Tel: +82-2-709-9241, Fax: +82-2-749-0499

E-mail: Ihthof@hanmail.net

ORCID: Jieon Go (https://orcid.org/0000-0002-4827-0929), Suyeon Park (https:// orcid.org/0000-0002-6391-557X), Kyeong Sik Kim (https://orcid.org/0000-00021463-8617), Min Chang Kang (https://orcid.org/0000-0002-0834-6060), Myong Hoon Ihn (https://orcid.org/0000-0002-9522-401X), Sangchul Yun (https://orcid. org/0000-0002-6321-4319), Sang Hyun Kim (https://orcid.org/0000-0002-03457044), Sung Hoon Hong (https://orcid.org/0000-0002-3279-3581), Jong Eun Lee (https://orcid.org/0000-0003-2938-4960), Sun Wook Han (https://orcid.org/00000002-0671-1212), Sung Yong Kim (https://orcid.org/0000-0002-2070-0915), Zisun Kim (https://orcid.org/0000-0002-1413-2800), Sung Mo Hur (https://orcid. org/0000-0001-8547-5635), Jihyoun Lee (https://orcid.org/0000-0003-00418717)

\section{Copyright (C) 2020 Korean Society of Surgical Oncology}

This is an Open Access article distributed under the terms of the Creative Commons Attribution Non-Commercial License (http://creativecommons.org/licenses/by-nc/4.0) which permits unrestricted non-commercial use, distribution, and reproduction in any medium, provided the original work is properly cited.

\section{INTRODUCTION}

Breast cancer treatment can accelerate bone loss and increase the incidence of osteoporosis and osteoporotic fracture [1,2]. Chemotherapy-induced amenorrhea and ovarian function suppression are related to the reduction in bone mineral density (BMD), and significant bone density loss and bone turnover have been reported in premenopausal patients with chemotherapy-induced amenorrhea [3]. The use of aromatase inhibitors has also been known to increase the risk of fracture [4]. Breast cancer survivors show an elevated incidence of fracture compared to the non-cancer population, and the risk increases with decreasing BMD [5]. Thus, several guidelines for breast cancer survivors recommend screening for osteoporosis, calcium and vitamin D supplementation, dietary education, and exercise [6,7] to mitigate this increased risk.

Osteoporosis and osteoporotic fracture are major concerns for breast cancer survivors that can have significant implications for quality of life; however, the risk of low bone density varies greatly 
between individuals. Exposure to estrogen is one of the risk factors for breast cancer, but it may also have a protective effect against osteoporosis [8]. Use of tamoxifen has been shown to have protective effects against bone loss in postmenopausal woman [9]. In addition, a population-based observational study on aromatase inhibitors and fracture risk in breast cancer survivors reported that the risk of fracture was comparable to that in the general population, after adjusting for age, baseline body mass index (BMI), osteoporosis medications, and other clinical factors [10].

Most research has focused on strategies for the treatment of breast cancer itself; investigation into the late and persistent effects of these treatments on the health status of long-term survivors is lacking. Chemotherapy, endocrine treatment, BMI, osteoporosis medications, health behaviors, and other clinical factors can offset the individual risk of bone loss, such that the real-world incidence of osteoporosis may differ from the results from clinical trials. In this study, we investigated the bone health status of long-term breast cancer survivors who had been treated for more than 5 years, compared to that in a non-cancer population, and evaluated treatment-related factors that have the potential to influence bone density.

\section{METHODS}

\section{Study population}

Patients from two tertiary healthcare institutions who had been diagnosed with breast cancer more than 5 years previously were enrolled from December 1, 2014, to November 30, 2015. To obtain a non-cancer control group for comparison, we retrieved data from the Korea National Health and Nutrition Examination Survey (KNHANES) during the study period (2014-2015) and selected control data at a case/control ratio of 1:4 based on age and survey year through propensity score matching. Individuals who were selected for the non-cancer control group had no recorded history of breast cancer according to the survey. The survey collected personal information on age (at the time of survey), residence, economic status, menopausal status, pregnancy, breastfeeding, body weight, BMI, family history of osteoporosis, and family history of fracture or rheumatoid arthritis, as well as clinical data including vitamin D levels and BMD. The survey additionally included a lifestyle questionnaire on fracture history, physical activity, smoking, alcohol intake, and nutritional supplementation. Individuals who worked out at for least 30 minutes five times a week were classified into the high physical activity group; those who drank alcohol less than once a month were included into the low alcohol consumption group. Smoking status was categorized as current smoker and past or nonsmoker. For the breast cancer patients who agreed to participate in the study, we obtained matching data from the KNHANES survey, as well as the clinical records regarding breast cancer status, including stage, pathologic results, and treatment.

We used the World Health Organization (WHO) guidelines. Osteoporosis is defined as a T-score below -2.5 based on BMD measurement, and osteopenia is defined as a T-score between -1.0 and -2.5 . We collected data on the lumbar spine and femur neck BMD and compared the incidences of osteoporosis and osteopenia between the two groups, along with the factors that can affect the development of low bone density. We additionally assessed the subsequent risk of osteoporotic fracture over a 10-year period in breast cancer survivors using a Fracture Risk Assessment Tool (FRAX; University of Sheffield, Sheffield, UK). This tool was developed by the WHO for predicting fracture risk using patient information such as age, body weight, height, previous fracture history, family history of osteoporosis and fracture, smoking history, and rheumatoid arthritis. This study received approval from the Institutional Review Board of Soonchunhyang University Hospital (IRB No. SCH 2015-10-019).

\section{Statistical analysis}

The chi-square test or Fisher exact test were used for the analysis of nominal variables. Continuous variables that were normally distributed, such as BMD of the lumbar spine and femur neck, were analyzed using the Student t-test. Variables that were not normally distributed, such as age, weight, BMI, age at the first pregnancy, and vitamin D level, were evaluated using the Mann-Whitney U test. Comparison of the incidence of osteoporosis between breast cancer survivors and non-cancer controls was performed using the Student t-test and Fisher exact test after adjusting for variables using the generalized estimating equation (GEE) by BMI. Factors affecting BMD were determined using the same procedures. The variables were assessed by GEE and adjusted for age and BMI. A P-value $<0.05$ was considered statistically significant. Statistical analyses were performed using SPSS 21.0 (IBM Corp., Armonk, NY, USA).

\section{RESULTS}

\section{Baseline characteristics of the study population}

A total of 74 long-term breast cancer survivors and 296 agematched non-cancer controls were included in the study. The mean age was $54.0 \pm 5.6$ in the breast cancer group and $54.3 \pm 6.7$ in the non-cancer control group $(\mathrm{P}=0.864)$. In the breast cancer survivor group, 30 patients (40.5\%) were premenopausal at diagnosis, which was significantly more than the 230 (77.7\%) pre- 
Table 1. Baseline characteristics of participants and clinical fetures of breast cancer survivors

\begin{tabular}{|c|c|c|c|}
\hline Characteristics & $\begin{array}{l}\text { Breast cancer } \\
\text { survivors }\end{array}$ & $\begin{array}{l}\text { Non-cancer } \\
\text { controls }\end{array}$ & P-value \\
\hline Age (yr) & $54.0 \pm 5.6$ & $54.3 \pm 6.7$ & 0.864 \\
\hline Residence & & & 0.034 \\
\hline Metropolitan & $22(29.7)$ & $129(43.6)$ & \\
\hline Provincial & $52(70.3)$ & $167(56.4)$ & \\
\hline Menopausal status & & & $<0.001$ \\
\hline Premenopausal & $28(37.8)$ & 75 (25.3) & \\
\hline Postmenopausal & $44(59.5)$ & $30(10.1)$ & \\
\hline Unknown & $2(2.7)$ & $191(64.5)$ & \\
\hline Pregnancy & & & $<0.001$ \\
\hline Ever & $57(77.0)$ & $232(78.4)$ & \\
\hline Never & $7(9.5)$ & $8(2.7)$ & \\
\hline Unknown & $10(13.5)$ & 56 (18.9) & \\
\hline Age of first pregnancy (yr) & $26.6 \pm 4.0$ & $21.1 \pm 7.1$ & $<0.001$ \\
\hline Breastfeeding & & & 0.033 \\
\hline Ever & 39 (70.9) & $164(83.7)$ & \\
\hline Never & $16(29.1)$ & $32(16.3)$ & \\
\hline Body weight (kg) & $58.3 \pm 9.0$ & $63.3 \pm 13.2$ & 0.016 \\
\hline BMI $\left(\mathrm{kg} / \mathrm{m}^{2}\right)$ & $23.7 \pm 3.8$ & $26.0 \pm 5.5$ & 0.003 \\
\hline Family history of osteoporosis & & & 0.524 \\
\hline Yes & $10(14.3)$ & $19(17.9)$ & \\
\hline No & $60(85.7)$ & $87(82.1)$ & \\
\hline \multicolumn{4}{|l|}{ Family history of fracture } \\
\hline Yes & $3(4.2)$ & - & \\
\hline No & $68(95.8)$ & - & \\
\hline Rheumatoid arthritis & & & 0.103 \\
\hline Yes & $1(1.4)$ & $15(6.0)$ & \\
\hline No & 73 (98.6) & $233(94.0)$ & \\
\hline \multicolumn{4}{|c|}{ Clinical information of breast cancer } \\
\hline Stage & & & - \\
\hline I & $29(39.7)$ & - & \\
\hline$\|$ & $33(45.2)$ & - & \\
\hline III & $11(15.1)$ & - & \\
\hline Type of surgery & & & - \\
\hline Total mastectomy & $21(28.8)$ & - & \\
\hline Partial mastectomy & $52(71.2)$ & - & \\
\hline Chemotherapy & & & - \\
\hline Yes & $60(82.2)$ & - & \\
\hline No & $13(17.8)$ & - & \\
\hline HER-2 targeted treatment & & & - \\
\hline Yes & $14(18.9)$ & - & \\
\hline No & $60(81.1)$ & - & \\
\hline Radiotherapy & & & - \\
\hline Yes & $57(77)$ & - & \\
\hline No & $17(23)$ & - & \\
\hline
\end{tabular}

(Continued to the next)
Table 1. Continued

\begin{tabular}{lccc}
\hline Characteristics & $\begin{array}{c}\text { Breast cancer } \\
\text { survivors }\end{array}$ & $\begin{array}{c}\text { Non-cancer } \\
\text { controls }\end{array}$ & P-value \\
\hline Endocrine treatment & $69(93.2)$ & - & - \\
$\quad$ Yes & $58(89.2)$ & - & \\
$\quad$ Tamoxifen & $7(10.8)$ & & \\
Aromatase inhibitor & $5(6.8)$ & - \\
No & $74(100)$ & $296(100)$ \\
\hline
\end{tabular}

Values are presented as mean \pm standard deviation or number (\%).

BMI, body mass index; HER-2, human epidermal growth factor receptor 2.

menopausal individuals in the control group $(\mathrm{P}<0.001)$. Age at the first pregnancy was also significantly different between the groups: patients in the breast cancer survivor group were older at the first pregnancy $(26.6 \pm 4.0$ years) than individuals in the control group $(21.1 \pm 7.1$ years $)(\mathrm{P}<0.001)$. Breastfeeding was significantly more prevalent in the non-cancer control group than in the breast cancer survivor group $(\mathrm{P}=0.033)$.

Mean body weight and BMI were both significantly higher in the non-cancer control group than in the breast cancer survivor group. The mean body weight was $58.3 \pm 9.0 \mathrm{~kg}$ in the breast cancer survivor group and $63.3 \pm 13.2 \mathrm{~kg}$ in the control group $(\mathrm{P}=$ 0.016 ), while the BMI was $23.7 \pm 3.8 \mathrm{~kg} / \mathrm{m}^{2}$ in breast cancer survivor group and $26.02 \pm 5.5 \mathrm{~kg} / \mathrm{m}^{2}$ in the control group $(\mathrm{P}=0.003)$. Family history of osteoporosis did not differ between the breast cancer survivor (14.3\%) and non-cancer control (17.9\%) groups $(\mathrm{P}=0.524)$. We identified one $(1.4 \%)$ breast cancer survivor with a history of rheumatoid arthritis, and no individual in the control group had a history of rheumatoid arthritis.

In the breast cancer survivor group, $82.2 \%$ of patients $(n=60)$ received chemotherapy and $93.2 \%$ of patients $(n=69)$ were treated with endocrine therapy. Among these, 89.2\% received tamoxifen and $10.8 \%$ received aromatase inhibitors (Table 1).

\section{Result of BMD measurement and incidence of fracture}

Fig. 1 shows the overall bone health status of each group. Among breast cancer survivors, $34.2 \%$ of patients had normal BMD; this proportion was lower than that in the non-cancer control group (53.4\%). Osteopenia was noted in $50.7 \%$ of breast cancer survivors, and $15.1 \%$ of patients had osteoporosis. In the non-cancer control group, 35.8\% of individuals had osteopenia and $10.8 \%$ had osteoporosis $(\mathrm{P}=0.028)$. The mean $\mathrm{BMD}$ of the lumbar spine in breast cancer survivors was $-1.30 \pm 1.25 \mathrm{~g} / \mathrm{cm}^{2}$, compared to $-0.94 \pm 1.32$ $\mathrm{g} / \mathrm{cm}^{2}$ in non-cancer controls (Table 2). The mean BMD of the femur neck was $-1.11 \pm 1.14 \mathrm{~g} / \mathrm{cm}^{2}$ in the breast cancer survivor 
group, compared to $-0.90 \pm 0.97 \mathrm{~g} / \mathrm{cm}^{2}$ in the control group $(\mathrm{P}=0.028)$. Serum vitamin $\mathrm{D}$ levels were higher in the breast cancer group than in the control group $(\mathrm{P}<0.001)$. There was no dif-

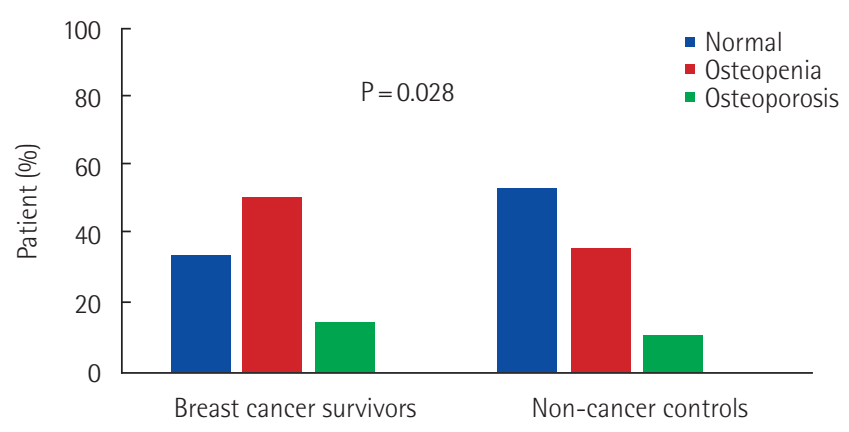

Fig. 1. Bone mineral density status in long-term breast cancer survivors compared to non-cancer controls. ference between the two groups in the incidence of fracture; breast cancer survivors had a fracture incidence of $2.7 \%$, whereas non-cancer controls had a fracture incidence of $9.2 \%(\mathrm{P}=0.146)$. We performed additional analyses after adjustment for BMI. Femur BMD was significantly lower in breast cancer survivors after adjustment $(\mathrm{P}=0.028)$, while serum vitamin $\mathrm{D}$ levels were consistently higher in breast cancer survivors after adjustment $(\mathrm{P}<0.001)$. There was no difference in the incidence of fracture between the two groups $(\mathrm{P}=0.130)$.

Factors related to low bone density, including osteopenia and osteoporosis, in breast cancer survivors after adjustment for age and BMI are described in Table 3. In patients with osteopenia, proportion of previous chemotherapy and endocrine therapy was higher, however, the difference was not statistically significant. Few patients were current smokers or drank alcohol; therefore, we were

Table 2. Bone health status in breast cancer survivors and non-cancer controls

\begin{tabular}{lcrc}
\hline Variable & Breast cancer survivors & Non-cancer controls & P-value \\
\hline Bone mineral density $\left(\mathrm{g} / \mathrm{cm}^{2}\right)$ & & & \\
Lumbar & $-1.30 \pm 1.25$ & $-0.94 \pm 1.32$ & 0.054 \\
Hip & $-1.11 \pm 1.14$ & $-0.90 \pm 0.97$ & 0.164 \\
Serum vitamin D $(\mathrm{ng} / \mathrm{mL})$ & $29.1 \pm 15.2$ & $17.6 \pm 6.3$ & $<.028$ \\
Incidence of fracture & & & $<(9.2)$ \\
Yes & $2(2.7)$ & $59(90.8)$ & 0.146 \\
No & $72(97.3)$ & $296(100)$ & 0.130 \\
Total & $74(100)$ & 501 & \\
\hline
\end{tabular}

Values are presented as mean \pm standard deviation or number (\%).

a)P-value by generalized estimating equation and adjusted by body mass index.

Table 3. Factors associated to osteopenia loss in breast cancer survivors

\begin{tabular}{|c|c|c|c|c|c|c|c|}
\hline Factor & & $\begin{array}{c}\text { Osteopenia } \\
\text { (lumbar) }\end{array}$ & OR $(95 \% \mathrm{Cl})$ & P-value & $\begin{array}{l}\text { Osteopenia } \\
\text { (femur) }\end{array}$ & OR $(95 \% \mathrm{Cl})$ & P-value ${ }^{a}$ \\
\hline \multirow[t]{2}{*}{ Chemotherapy } & Yes & $38(84.4)$ & $0.287(0.044-1.854)$ & 0.190 & $32(78)$ & $1.424(0.283-7.169)$ & 0.668 \\
\hline & No & $7(15.6)$ & Reference & & $9(22)$ & Reference & \\
\hline \multirow[t]{2}{*}{ Endocrine treatment } & Yes & 42 (91.3) & $5.167(0.496-53.823)$ & 0.170 & $38(90.5)$ & $5.337(0.565-50.423)$ & 0.144 \\
\hline & No & $4(8.7)$ & Reference & & $4(9.5)$ & Reference & \\
\hline \multirow[t]{2}{*}{ Alcohol consumption } & Yes & $1(2.2)$ & - & NA & 0 & - & NA \\
\hline & No & 45 (97.8) & - & - & $42(100)$ & - & - \\
\hline \multirow[t]{2}{*}{ Smoking } & Yes & $3(6.7)$ & - & NA & $3(7.3)$ & - & NA \\
\hline & No & 42 (93.3) & - & - & 38 (92.7) & - & - \\
\hline \multirow[t]{2}{*}{ Exercise } & Yes & $37(80.4)$ & $0.866(0.220-3.407)$ & 0.837 & 31 (73.8) & $3.266(0.777-13.734)$ & 0.106 \\
\hline & No & $9(19.6)$ & Reference & & $11(26.2)$ & Reference & \\
\hline \multirow[t]{2}{*}{ Vitamin D supplements } & Yes & 35 (76.1) & $0.517(0.154-1.742)$ & 0.287 & $33(78.6)$ & $0.457(0.147-1.426)$ & 0.177 \\
\hline & No & $11(23.9)$ & Reference & & $9(21.4)$ & Reference & \\
\hline \multirow[t]{2}{*}{ Calcium supplements } & Yes & $33(71.7)$ & $0.429(0.132-1.392)$ & 0.159 & $30(71.4)$ & $0.462(0.153-1.394)$ & 0.170 \\
\hline & No & $13(28.3)$ & Reference & & $12(28.6)$ & Reference & \\
\hline
\end{tabular}

$\mathrm{OR}$, odds ratio; $\mathrm{Cl}$, confidence interval; $\mathrm{NA}$, not available.

a)P-value by generalized estimating equation and adjusted by age and body mass index. 


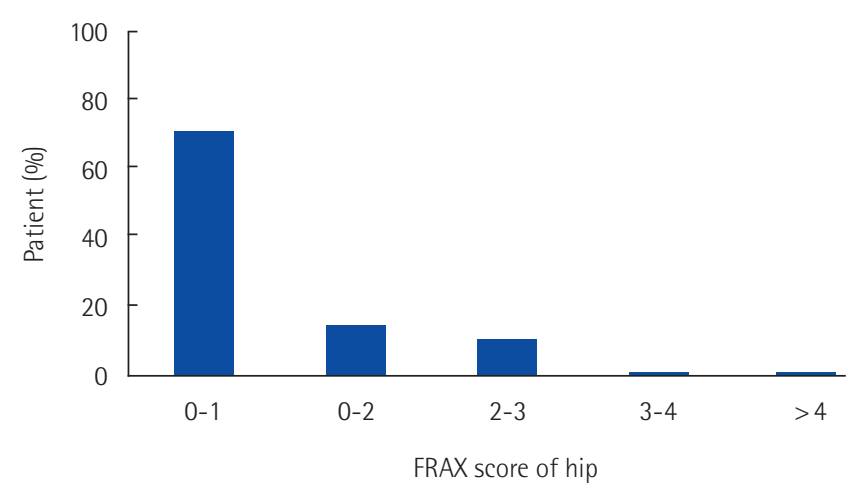

Fig. 2. Ten-year risk assessment of osteoporotic femur fracture using Fracture Risk Assessment Tool (FRAX) score.

unable to evaluate these factors. There was no significant difference in bone density with respect to the level of physical exercise, vitamin D supplementation, or calcium supplementation.

The FRAX score of breast cancer survivors is shown in Fig. 2. The 10-year risk of femur neck fracture was less than $1 \%$ in $71.62 \%$ of survivors. Almost all $(\mathrm{n}=72 ; 97.29 \%)$ of the breast cancer patients had a 10-year risk lower than $3 \%$.

\section{DISCUSSION}

In this study, we found that the incidence of fracture was comparable between long-term breast cancer survivors and non-cancer agematched controls. Although BMD of the femur neck was significantly lower in breast cancer survivors, the actual risk of fracture was similar in both groups. We found that the 10-year risk for femur neck fracture was less than $3 \%$ in most survivors, while in example case of age 55 years with femur BMD $-1.0 \mathrm{~g} / \mathrm{cm}^{2}$, and BMI $24 \mathrm{~kg} / \mathrm{m}^{2}, 10-$ year probability of hip fracture assumed by FRAX presents $0.3 \%$ in the absence of additional risk factors.

With the increase in the number of long-term breast cancer survivors, there have been several reports on their health status. After treatment for breast cancer, lymphedema, osteoporosis, and heart disease can occur. Ovarian dysfunction can also occur due to endocrine therapy and chemotherapy, leading to bone loss. To prevent osteoporosis, guidelines recommend bisphosphonate therapy with calcium and vitamin D supplementation and monitoring of BMD as risk assessment tool prior to the administration of aromatase inhibitors [11-13]. A previous study reported at least one secondary cause of bone loss other than cancer-related treatment in $78 \%$ of patients in the breast cancer group and $77 \%$ of individuals in the non-breast cancer group, and the most common causes of osteopenia were vitamin D deficiency, idiopathic hypercalciuria, and primary hyperparathyroidism [14]. Adjuvant treatment for breast cancer can affect bone density, and while there have been several studies encompassing the treatment periods, there are limited data on the status of long-term survivors and on whether it is possible to restore low bone density after initial adjuvant therapy.

Most breast cancer survivors included in this study were treated with chemotherapy or endocrine therapy, which can induce ovarian dysfunction, leading to early menopause [15]. Estrogen is involved in the preservation of bone mass and block bone resorption [16]. Chemotherapeutic agents may suppress ovarian function and promote bone loss in premenopausal women [17]. Tamoxifen, one of the most frequently prescribed medications in endocrine treatment, can cause osteoporosis by inducing estrogen deficiency in premenopausal women, while having a protective effect on postmenopausal patients. The use of aromatase inhibitors is also related to an increased incidence of fracture [18]. In this study, breast cancer survivors had a higher rate of premenopausal status than the non-cancer controls; thus, it is possible that premenopausal tamoxifen exposure influenced the reduction in bone density.

Although the breast cancer survivor group displayed a low BMD compared to the non-cancer controls, vitamin D levels in this group were significantly higher. If the level of vitamin D is low, bone mineralization goes defective and results in low bone mass [19]. Vitamin D deficiency is known as a surrogate risk factor for breast cancer [20], and vitamin D supplementation is associated with improved outcomes in colorectal cancer $[21,22]$. However, it has yet not been established whether routine supplementation with vitamin $\mathrm{D}$ can improve survival outcomes in breast cancer. Regardless of the improvement in cancer-related survival, appropriate vitamin $\mathrm{D}$ and calcium supplementation are important for healthcare in breast cancer survivors [23,24]. In the two institutions from which our cohort was selected, evaluation of vitamin D levels was left to the preference of the physician; therefore, most long-term breast cancer survivors who had low vitamin D levels had been recommended to take vitamin D and calcium supplements.

One patient in this study with a FRAX score of 4 had the highest risk of osteoporotic fracture; she was diagnosed before menopause and underwent partial mastectomy followed by radiation therapy, chemotherapy, and endocrine therapy. She was diagnosed with osteoporosis but did not experience osteoporotic fracture even with a low BMD. Her vitamin D levels were routinely checked, and she received vitamin $\mathrm{D}$ and calcium supplementation and bisphosphonate treatment; this demonstrates the importance of optimal supportive care.

In the present study, we evaluated long-term survivors who were diagnosed with breast cancer and treated with multiple treatments, who may have altered their lifestyle during treatment, or who may have been under a physician's care for the late consequences of ad- 
juvant treatment. We compared these patients with real-world data from the KNHANES, effectively establishing an age-matched control group rather than using hospital-based controls. Our findings did not reveal significant risk factors for low bone density related to previous treatment history. It is possible that adjuvant treatment may not have long-term effects or that supportive care can offset the effects of adjuvant treatment, which needs further evaluation.

This study has several limitations. The questionnaire was dependent on patient recall and can reflect lifestyle modifications made after a diagnosis of osteoporosis in both breast cancer patients and the non-cancer control group; thus, it is difficult to determine which factors influence each other. Moreover, we were not able to retrieve data on the family history of fractures or corticosteroid use, which are essential for calculating the FRAX score in non-cancer population. Although we investigated the incidence of fracture, we were unable to determine whether osteoporosis was the cause of fracture. Finally, there were only a few long-term survivors who had been diagnosed for more than 5 years; thus, the number of patients included in this study was relatively small.

In conclusion, we found low bone density in long-term breast cancer survivors compared to that in the general population; however, the incidence of fractures between the groups were comparable. Our findings indicate that beyond cancer treatment, appropriate survivorship care is recommended to improve bone health in long-term breast cancer survivors.

\section{CONFLICT OF INTEREST}

No potential conflict of interest relevant to this article was reported.

\section{ACKNOWLEDGMENTS}

The present research has been supported by Korea Breast Cancer Foundation and the Soonchunhyang University Research Fund.

We appreciate the contributions of the Study of Multi-disciplinARy Teamwork of breast cancer survivorSHIP (SMARTSHIP) Group to this study: Ku Sang Kim, Nam Hyong Kim, Zisun Kim, Eun Jung Shim, Min Hyuk Lee, Jung Eun Lee, So Yeon Jung, Ji Hyung Cho, Yu Seok Kim, Jun Won Min, Sekyung Lee, Il Kyun Lee, Yong Jin Choi, Hyuk Jae Shin, Sung Min Park, Hyeng-Gon Moon, Jong Eon Lee, Seho Park, Byong Ju Chae, Il Yong Chun, Jong Won Lee, Hyun Jo Youn, and Ho Hur.

\section{REFERENCES}

1. Bruyere O, Bergmann P, Cavalier E, Gielen E, Goemaere S, Kaufman JM, et al. Skeletal health in breast cancer survivors. Matu- ritas 2017;105:78-82.

2. Peppone LJ, Mustian KM, Rosier RN, Carroll JK, Purnell JQ, Janelsins MC, et al. Bone health issues in breast cancer survivors: a Medicare Current Beneficiary Survey (MCBS) study. Support Care Cancer 2014;22:245-51.

3. Hadji P, Ziller M, Maskow C, Albert U, Kalder M. The influence of chemotherapy on bone mineral density, quantitative ultrasonometry and bone turnover in pre-menopausal women with breast cancer. Eur J Cancer 2009;45:3205-12.

4. Hadji P. Aromatase inhibitor-associated bone loss in breast cancer patients is distinct from postmenopausal osteoporosis. Crit Rev Oncol Hematol 2009;69:73-82.

5. Body JJ. Increased fracture rate in women with breast cancer: a review of the hidden risk. BMC Cancer 2011;11:384.

6. Coleman R, Body JJ, Aapro M, Hadji P, Herrstedt J; ESMO Guidelines Working Group. Bone health in cancer patients: ESMO Clinical Practice Guidelines. Ann Oncol 2014;25 Suppl 3:iii124-37.

7. Van Poznak CH, Von Roenn JH, Temin S. American society of clinical oncology clinical practice guideline update: recommendations on the role of bone-modifying agents in metastatic breast cancer. J Oncol Pract 2011;7:117-21.

8. Lamont EB, Lauderdale DS. Low risk of hip fracture among elderly breast cancer survivors. Ann Epidemiol 2003;13:698-703.

9. Powles TJ, Hickish T, Kanis JA, Tidy A, Ashley S. Effect of tamoxifen on bone mineral density measured by dual-energy x-ray absorptiometry in healthy premenopausal and postmenopausal women. J Clin Oncol 1996;14:78-84.

10. Leslie WD, Morin SN, Lix LM, Niraula S, McCloskey EV, Johansson $\mathrm{H}$, et al. Fracture risk in women with breast cancer initiating aromatase inhibitor therapy: a registry-based cohort study. Oncologist 2019;24:1432-8.

11. Agrawal S. Late effects of cancer treatment in breast cancer survivors. South Asian J Cancer 2014;3:112-5.

12. Hill DA, Horick NK, Isaacs C, Domchek SM, Tomlinson GE, Lowery JT, et al. Long-term risk of medical conditions associated with breast cancer treatment. Breast Cancer Res Treat 2014;145:233-43.

13. Lash TL, Thwin SS, Yood MU, Geiger AM, Bosco J, Quinn VP, et al. Comprehensive evaluation of the incidence of late effects in 5-year survivors of breast cancer. Breast Cancer Res Treat 2014;144:64363.

14. Camacho PM, Dayal AS, Diaz JL, Nabhan FA, Agarwal M, Norton JG, et al. Prevalence of secondary causes of bone loss among breast cancer patients with osteopenia and osteoporosis. J Clin Oncol 2008;26:5380-5.

15. Kalder M, Hadji P. Breast cancer and osteoporosis: management of cancer treatment-induced bone loss in postmenopausal women with breast cancer. Breast Care (Basel) 2014;9:312-7. 
16. Van Poznak C, Sauter NP. Clinical management of osteoporosis in women with a history of breast carcinoma. Cancer 2005;104:44356.

17. Bruning PF, Pit MJ, de Jong-Bakker M, van den Ende A, Hart A, van Enk A. Bone mineral density after adjuvant chemotherapy for premenopausal breast cancer. Br J Cancer 1990;61:308-10.

18. Taxel P, Choksi P, Van Poznak C. The management of osteoporosis in breast cancer survivors. Maturitas 2012;73:275-9.

19. Sadat-Ali M, Al Elq AH, Al-Turki HA, Al-Mulhim FA, Al-Ali AK. Influence of vitamin $\mathrm{D}$ levels on bone mineral density and osteoporosis. Ann Saudi Med 2011;31:602-8.

20. Atoum M, Alzoughool F. Vitamin D and breast cancer: latest evidence and future steps. Breast Cancer (Auckl) 2017;11:1178223417749816.

21. Mondul AM, Weinstein SJ, Layne TM, Albanes D. Vitamin D and cancer risk and mortality: state of the science, gaps, and challenges. Epidemiol Rev 2017;39:28-48.

22. Wesselink E, Kok DE, Bours MJL, de Wilt JHW, van Baar H, van Zutphen M, et al. Vitamin D, magnesium, calcium, and their interaction in relation to colorectal cancer recurrence and all-cause mortality. Am J Clin Nutr 2020;111:1007-17.

23. Prentice RL, Pettinger MB, Jackson RD, Wactawski-Wende J, Lacroix AZ, Anderson GL, et al. Health risks and benefits from calcium and vitamin D supplementation: Women's Health Initiative clinical trial and cohort study. Osteoporos Int 2013;24:567-80.

24. Santen RJ, Stuenkel CA, Davis SR, Pinkerton JV, Gompel A, Lumsden MA. Managing menopausal symptoms and associated clinical issues in breast cancer survivors. J Clin Endocrinol Metab 2017; 102:3647-61. 